Draft Version June 15, 2022

Preprint typeset using $\mathrm{LAT}_{\mathrm{E}} \mathrm{X}$ style emulateapj v. 12/16/11

\title{
GALAXY-SCALE GRAVITATIONAL LENS CANDIDATES FROM THE HYPER SUPRIME-CAM IMAGING SURVEY AND THE GALAXY AND MASS ASSEMBLY SPECTROSCOPIC SURVEY
}

\author{
James H. H. Chan ${ }^{1,2}$, Sherry H. Suyu ${ }^{3,2}$, Anupreeta More ${ }^{4}$, Masamune Oguri ${ }^{5,6,7}$, Tzihong Chiueh ${ }^{1,8,9}$, Jean \\ Coupon $^{10}$, Bau-Ching Hsien ${ }^{2}$, Yutaka Komiyama ${ }^{11,12}$, Satoshi MiYazaki ${ }^{11,12}$, Hitoshi Murayama ${ }^{5,11,12}$, Atsushi \\ J. Nishizawa ${ }^{15}$, Paul Price ${ }^{16}$, Philip J. Tait ${ }^{17}$, Tsuyoshi Terai ${ }^{17}$, Yousuke Utsumi ${ }^{18}$, Shiang-Yu Wang ${ }^{2}$ \\ Draft version June 15, 2022
}

\begin{abstract}
We present a list of galaxy-scale lens candidates including a highly probable interacting galaxy-scale lens in the Hyper Suprime-Cam (HSC) imaging survey. We combine HSC imaging with the blendedspectra catalog from the Galaxy And Mass Assembly (GAMA) survey to identify lens candidates, and use lens mass modeling to confirm the candidates. There are 45 matches between the HSC S14A_0b imaging data release and the GAMA catalog. We separate lens and lensed arcs using color information, and exclude those candidates with small image separations $\left(<1.0^{\prime \prime}\right.$, estimated with the lens/source redshifts from the GAMA survey) that are not easily resolved with ground-based imaging. After excluding these, we find ten probable lens systems. There is one system with an interacting galaxy pair, HSC J084928+000949, that has a valid mass model. We predict the total mass enclosed by the Einstein radius of $\sim 0.72^{\prime \prime}(\sim 1.65 \mathrm{kpc})$ for this new expected lens system to be $\sim 10^{10.59} M_{\odot}$. Using the photometry in the grizy bands of the HSC survey and stellar population synthesis modeling with a Salpeter stellar initial mass function, we estimate the stellar mass within the Einstein radius to be $\sim 10^{10.46} M_{\odot}$. We thus find a dark matter mass fraction within the Einstein radius of $\sim 25 \%$. Further spectroscopy or high-resolution imaging would allow confirmation of the nature of these lens candidates. The particular system with the interacting galaxy pair, if confirmed, would provide an opportunity to study the interplay between dark matter and stars as galaxies build up through hierarchical mergers.
\end{abstract}

\section{INTRODUCTION}

d00222002@ntu.edu.tw

${ }^{1}$ Department of Physics, National Taiwan University, Taipei 10617, Taiwan

${ }^{2}$ Institute of Astronomy and Astrophysics, Academia Sinica, P.O. Box 23-141, Taipei 10617, Taiwan

${ }^{3}$ Max-Planck-Institut für Astrophysik, Karl-SchwarzschildStr. 1, 85741 Garching, Germany

${ }^{4}$ Kavli IPMU (WPI), UTIAS, The University of Tokyo, Kashiwa, Chiba 277-8583, Japan

${ }^{5}$ Kavli Institute for the Physics and Mathematics of the Universe (Kavli IPMU, WPI), University of Tokyo, 5-1-5 Kashiwanoha, Kashiwa-shi, Chiba 277-8583, Japan

${ }^{6}$ Research Center for the Early Universe, University of Tokyo, 7-3-1 Hongo, Bunkyo-ku, Tokyo 113-0033, Japan

${ }^{7}$ Department of Physics, University of Tokyo, 7-3-1 Hongo, Bunkyo-ku, Tokyo 113-0033, Japan

${ }^{8}$ Institute of Astrophysics, National Taiwan University, 10617 Taipei, Taiwan

${ }_{9}$ Center for Theoretical Sciences, National Taiwan University, 10617 Taipei, Taiwan

${ }^{10}$ Astronomical Observatory of the University of Geneva, ch. d'Ecogia 16, CH-1290 Versoix, Switzerland

${ }^{11}$ University of California, Berkeley, CA 94720, USA

12 Lawrence Berkeley National Laboratory, MS 50A-5104, Berkeley, CA 94720. USA

13 National Astronomical Observatory of Japan, 2-21-1 Osawa, Mitaka, Tokyo 181-8588, Japan

${ }_{14}$ Department of Astronomy, School of Science, SOKENDAI (The Graduate University for Advanced Studies), Mitaka, Tokyo 181-8588

${ }^{15}$ Institute for Advanced Research, Nagoya University, Furocho Chikusa Nagoya Aichi 4648602, Japan

${ }^{16}$ Princeton University Observatory, Peyton Hall, Princeton, NJ 08544, USA

${ }^{17}$ National Astronomical Observatory of Japan, 650 North A'ohoku Place, Hilo, HI 96720, USA

18 Hiroshima Astrophysical Science Center, Hiroshima University, 1-3-1 Kagamiyama, Higashi-Hiroshima, Hiroshima 7398526, Japan
Strong gravitational lensing is a powerful tool for measuring the mass distribution from galaxies to galaxy clusters. Understanding the interplay between dark matter and baryons at galactic/sub-galactic scales is crucial to study the formation and evolution of galaxies. Moreover, the signal from background source objects is magnified so we can make use of this information to probe the highredshift universe.

Strong lens systems are rare, however. A lensing phenomenon happens when a massive foreground deflector is sufficiently well aligned along the line of sight to background source galaxies. Searches for strongly lensed galaxies have been carried out extensively in large imaging and spectroscopic surveys, including the Sloan Lens ACS Survey (SLACS; e.g., Bolton et al. 2006), the CFHTLSI9] Strong Lensing Legacy Survey (SL2S; e.g., Cabanac et al. 2007; Gavazzi et al. 2012; More et al. 2012; Sonnenfeld et al. 2013a, b), the BOSS Emissionline Lens Survey (BELLS; e.g., Brownstein et al. 2012; Bolton et al. 2012), the HST Archive Galaxy-scale Gravitational Lens Search (HAGGLeS; Marshall et al. 2009), Herschel ATLAS (H-ATLAS; e.g., Negrello et al. 2010; González-Nuevo et al. 2012), and the South Pole Telescope (SPT; e.g., Vieira et al.|2013; Hezaveh et al.|2013). Through these galaxy-galaxy strong lens surveys, there are now a couple of hundreds of strong lenses with different source populations.

In this work, we start from all the candidates in the Galaxy And Mass Assembly (GAMA) blended spectra

19 Canada-France-Hawaii Telescope Legacy Survey. See http://www.cfht.hawaii.edu/Science/CFHLS/ and links therein tor a comprehensive description. 
catalog (Holwerda et al. 2015) and visually inspect their Hyper Suprime-Cam (HSC) images to prune the lens candidates further. With high image quality in the HSC survey, we can discern foreground lens and background source with CHITAH (Chan et al. 2015) using color information, and further reconstruct the lens mass distribution and lensed source surface brightness with the lens modeling software GLEE (Suyu \& Halkola 2010; Suvu et al. 2012). Our philosophy is to use lens modeling to confirm lens candidates (as advocated by, e.g., Marshall et al. 2009), since a lens system must be explainable by a physical lens mass model. Through lens mass modeling, we report ten probable lens candidates, and one of them is a highly probable interacting galaxyscale lens system.

This paper is organized as follows. In Section 2 we overview the observational data with spectroscopy and imaging. The procedure of lens examination is described in Section 3. We list probable lens candidates in Section 4. In Section 5. conclusions are drawn and future prospect is discussed.

\section{OBSERVATION}

We summarize the spectroscopic and imaging observations of the new lens candidates in GAMA and HSC surveys in Section 2.1 and Section 2.2 respectively.

\subsection{GAMA blended spectra catalog}

The GAMA survey has spectroscopic redshifts of 300,000 galaxies down to $r<19.8 \mathrm{mag}$ over 286 $\operatorname{deg}^{2}$ with the upgraded $2 \mathrm{dF}$ spectrograph AAOmega on the Anglo-Australian Telescope (Driver et al. 2009, 2011; Baldry et al. 2010). The fiber size is $2^{\prime \prime}$ in diameter projected on the sky (Hopkins et al. 2013). In the GAMA survey pipeline, Baldry et al. (2014) developed a fully automatic algorithm, AUTOZ, which measures redshift using a cross-correlation method for both absorption-line and emission-line features.

An object with blended spectra is likely to be a lens candidate. Holwerda et al. (2015) have classified the blended spectra by the best-fit passive/emissionline templates and have listed 280 objects whose features of blended spectra are separated by at least 600 $\mathrm{km} / \mathrm{s}$. They visually inspected the images of candidates in the Sloan Digital Sky Survey (SDSS) and Kilo Degreee Survey (KiDS) and obtained 104 lens candidates and 176 occulting galaxy pairs, similar to the search in Holwerda et al. (2007) in SDSS, supplementing eyeball searches in Galaxy Zoo (Keel et al. 2013). The catalog provides useful information for us to further classify some of the lens candidates based on the HSC imaging survey with better image quality.

\subsection{HSC imaging}

HSC is the new prime-focus camera on the 8.2-m Subaru telescope located at Mauna Kea, Hawaii. This new camera together with the large telescope provides uniform quality of images across a wide area of the sky, i.e., less affected by the vignetting and distortion around the edge of the field of view (FOV). The FOV of HSC is 1.5 deg in diameter (Mivazaki et al. 2012) which is ten times larger than the FOV of Suprime-Cam (Mivazaki et al. 2002). The HSC Subaru Strategic Program (SSP) survey consists of three layers with different depths: wide, deep and ultra-deep. The wide survey will cover $\sim 1400$ $\operatorname{deg}^{2}$ to $i \sim 25.8 \mathrm{mag}$ in grizy broad bands. We identify and model candidates in the internal early data release S14A_0b (XMM, GAMA09h, VVDS) of the HSC imaging survey.

The data are processed with hscPipe 3.4.1, a derivative of the the Large Synoptic Survey Telescope (LSST) pipeline (Ivezic et al. 2008; Axelrod et al. 2010), modified for use with Suprime-Cam and Hyper SuprimeCam. The photometric calibration is based on data obtained from the Panoramic Survey Telescope and Rapid Response System (Pan-STARRS) 1 imaging survey (Schlafly et al. 2012; Tonry et al. 2012; Magnier et al. 2013). We use HSC SSP data release S15A reduced with hscPipe 3.8.5, which was released near the final stages of our analysis, to update our lens models of the candidates identified in S14A_0b. The pixel scale of the reduced images in the data releases is $0.168^{\prime \prime}$. The seeing is $0.6^{\prime \prime}$ in the $i$-band and $0.68^{\prime \prime}-0.81^{\prime \prime}$ in others.

\section{METHOD}

To further confirm the lens candidates in the GAMA blended spectra catalog, we visually select objects based on their morphology and redshift information. Moreover, we try to reconstruct background sources via lens modeling. If a candidate is a lens system, its reconstructed source components should be well described by regular looking galaxies. In other words, we can confirm a system to be a lens if we can find a physically sensible model for the foreground lens mass distribution and the background source surface brightness.

\subsection{Selection via morphology and redshift information}

We start with the 280 objects from the GAMA blended spectra catalog. There are 45 objects in the footprint of the HSC early data release S14A_0b. We exclude two of them with incomplete HSC imaging data. In the remaining 43 objects, there are 14 objects identified as strong lens candidates in Holwerda et al. (2015). We note that these objects are categorized by Holwerda et al. (2015) as having a passive galaxy (PG) at a lower redshift near an emission-line galaxy (ELG) at a higher redshift. In addition to these types of "PG+ELG" blended spectra (foreground passive galaxy with background emissionline galaxy), there are the "PG+PG" (foreground and background passive galaxies), "ELG+PG" (foreground emission-line galaxy with background passive galaxy) and "E+E" (foreground and background emission-line galaxies) types identified by Holwerda et al. (2015) that could also be potential strong lenses. Therefore, we consider all the 43 galaxies from Holwerda et al. (2015) with $\mathrm{HSC}$ imaging in the rest of the paper.

We employ CHitaH (Chan et al. 2015) to disentangle lens and lensed-source components based on color information. Chitah combines two image cutouts, one from the bluer bands ( $g$ or $r$ ) and one from the redder bands $(z$ or $y$ ), to produce a "foreground lens image" and a "lensed arc image of the background source". CHITAH chooses the band with a narrower PSF from the two bluer bands, and similarly from the two redder bands. By selecting the cutouts with narrower PSFs, CHITAH produces the sharpest possible images of the lens and lensed arcs. We show in Figure1(c) and (d) an example of the lens light and lensed arc separation, respectively. 


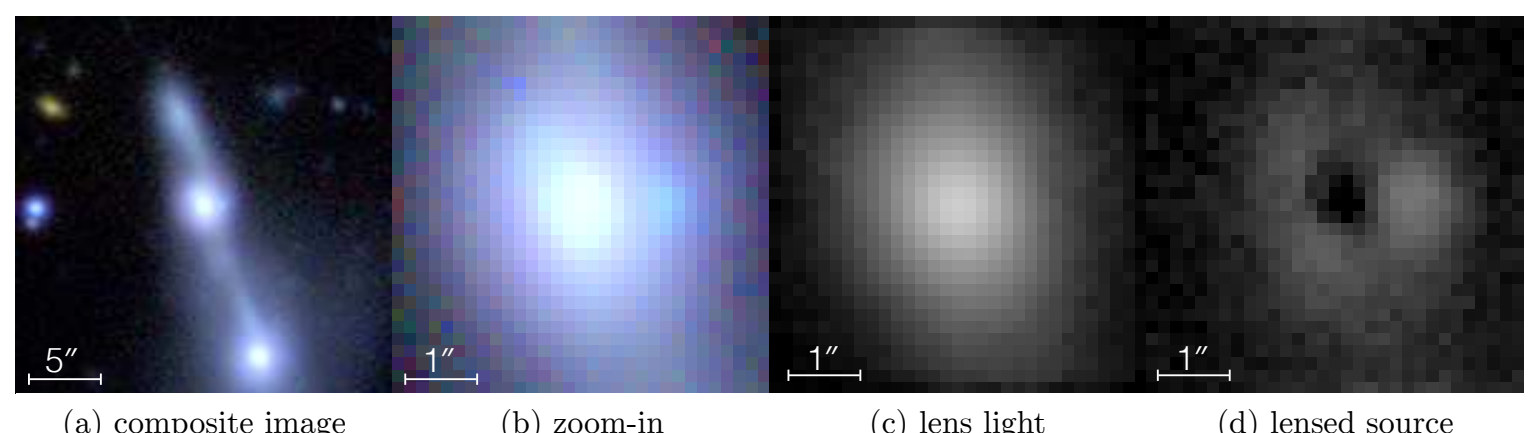
(a) composite image
(b) zoom-in
(c) lens light
(d) lensed source

FIG. 1. - The highly probable lens system from HSC survey: HSC J084928+000949. The riz composite cutout in $25^{\prime \prime} \times 25^{\prime \prime}$ shows clearly the interacting feature in panel (a). Panel (b) is the central $5^{\prime \prime} \times 5^{\prime \prime}$ region of the riz composite. CHITAH separates the light components in panel (b) into the lens light (panel (c)) and lensed arcs of the background source (panel (d)) using color information. The GAMA spectroscopic redshifts are $\left(z_{1}, z_{2}\right)=\left(0.128^{\mathrm{P}}, 0.603^{\mathrm{E}}\right)$, where $\mathrm{P}$ and E stand for "Passive galaxy" and "Emission-line galaxies", respectively.

Furthermore, Holwerda et al. (2015) also provided two galaxies' redshifts of each lens candidate which allow us to predict image separations, $\vartheta_{\text {sep }}$, with a fiducial velocity dispersion of $\sigma \sim 250 \mathrm{~km} / \mathrm{s}$,

$$
\vartheta_{\text {sep }}=4 \pi \frac{\sigma^{2} D_{\mathrm{LS}}}{c^{2} D_{\mathrm{S}}}
$$

where $D_{\mathrm{LS}}$ is the angular diameter distance between the lens and the source, and $D_{\mathrm{S}}$ is the angular distance between us and the source. Those lens candidates with lens/source redshift values that are too close are excluded since the image separations are too small $\left(\vartheta_{\text {sep }}<1.0^{\prime \prime}\right)$ and difficult to resolve in the HSC images. We also empirically rule out the candidates that have no lensed arc features and only 10 candidates remain. In other words, when the lensed-arc feature can be seen such as that shown in Figure11(d), we then try to model the lens mass distribution and source surface brightness of the system.

\subsection{Lens model}

To confirm the lensing nature of the 10 probable candidates, we use the lens modeling software GLEE, developed by A. Halkola and S. H. Suyu (Suyu \& Halkola 2010; Suyu et al. 2012), to fit the lens light and lensedsource components. Lens light components show stellar distributions of lens galaxies, which can be well described by Sérsic profiles (Sérsic 1963):

$$
I\left(\theta_{1}, \theta_{2}\right)=A \exp \left[-k\left(\left(\frac{\sqrt{\theta_{1}^{2}+\theta_{2}^{2} / q_{\mathrm{L}}^{2}}}{R_{\mathrm{eff}}}\right)^{1 / n_{\mathrm{s}}}-1\right)\right],
$$

where $\left(\theta_{1}, \theta_{2}\right)$ are image coordinates, $A$ is the amplitude, $k$ is a constant such that $R_{\text {eff }}$ is the effective (halflight) radius, $q_{\mathrm{L}}$ is the axis ratio, and $n_{\mathrm{s}}$ is the Sérsic index. To fit to the lens light image from CHITAH, we have as additional parameters the centroid $\left(\theta_{1 \mathrm{c}}, \theta_{2 \mathrm{c}}\right)$ and the position angle of the Sérsic distribution. For the lens galaxy's dimensionless surface mass density (the total mass distribution, including baryons and dark matter), we adopt pseudo-isothermal elliptic mass distribution (PIEMD; Kassiola \& Kovner 1993) with a vanishing core:

$$
\kappa\left(\theta_{1}, \theta_{2}\right)=\frac{r_{\text {ein }}}{2 \sqrt{\frac{\theta_{1}^{2}}{(1+\epsilon)^{2}}+\frac{\theta_{2}^{2}}{(1-\epsilon)^{2}}}},
$$

where $r_{\text {ein }}$ is the Einstein radius of the lens (that is related to the strength of the lens) and $\epsilon$ is the ellipticity that is related to the axis ratio $q$ by $\epsilon \equiv(1-q) /(1+q)$.

We first model the lens light produced in the colordifference image as shown in Figure 1(c) to obtain the Sérsic parameters, particularly the centroid and position angle. We then model the lensed arcs to constrain simultaneously the source surface brightness on a grid of pixels and the lens mass parameters of PIEMD. We adopt the curvature form of regularization on the source grid, and Gaussian priors on the centroid $\left(\sim 0.05^{\prime \prime}\right)$, the axis ratio $(\sim 0.1)$, and the position angle $(\sim 3 \mathrm{deg})$ of the PIEMD based on the fitted Sérsic parameters. We allow the Einstein radius to vary with uniform prior. We use GLEE to confirm the lensing nature for those systems that have sensible source surface brightness and lens mass model parameters, as detailed next.

\section{GRAVITATIONAL LENS CANDIDATES FROM HSC SURVEY}

Of the ten candidates that show arc-like features in Section 3.1. we cannot obtain reasonable lens models with GLEE for nine of them, due to e.g. $r_{\text {ein }}$ that are too small/large or inverted sources that appear unphysical as a result of the limited number of image pixels. We list these nine lens candidates in Section 4.1. We consider one candidate that is the most probable since we can obtain a sensible lens model with GLEE and discuss it in Section 4.2. The remaining 33 objects classified as possible lenses or non-lenses are listed in Appendix A.

\subsection{Probable lens candidates}

The lens candidates are listed in Figure 2 and Table 1 for future confirmation. Except for HSC J084928+000949 which we discuss in more detail in Section 4.2 we classify the remaining nine systems as probable lens candidates. Five of these nine probable lens candidates were previously identified by Holwerda et al. (2015) as possible strong lenses with foreground passive lens galaxies and background emission-line source galaxies (i.e., the six objects in Figure 2 with "P" and "E" flagged on $z_{1}$ and $z_{2}$, respectively).

We identify a probable binary lens system in J022511-045033. J084552+011156 and J084406+013853 are likely disky lenses. Some of the can- 
didates are low-redshift lenses, such as J084202+010115, J085005+021740 and J085029+001533. These candidates are worth inspecting with higher-resolution imaging, as they complement nicely the existing samples of lenses at higher redshifts, e.g., SLACS, SL2S and BELLS.

\subsection{A promising interacting-galaxy lens candidate from $H S C$ survey}

In this work, we identify one very promising lens candidate, HSC J084928+000949, from HSC survey via lens modeling. The result is shown in Figure 1 . CHITAH separates lens light and lensed arcs using $g$-band cutout instead of $r$-band cutout which has bad pixels, even though the $r$-band PSF is narrower than that of the $g$-band. The coordinate is $(\mathrm{RA}, \mathrm{DEC})=(132.36771,0.16360)$. We also notice that this candidate is an interacting galaxypair which is an important aspect of galaxy evolution (e.g., Hopkins et al. 2006; Lotz et al. 2008). Gravitational lensing provides an independent constraint on dark matter distribution. Hence, it can help us to study the interplay between dark matter and baryonic components (e.g., Barnabè et al. 2011; Sonnenfeld et al. 2015).

We also check the GAMA spectra shown in Figure 3 (Liske et al. 2015) to verify that it is blended of two galaxies at different redshifts. The two galaxy types are emission line galaxy $\left(z_{2} \sim 0.603\right)$ and passive galaxy $\left(z_{1} \sim 0.128\right)$ - in fact, this system was previously identified by Holwerda et al. (2015) as a lens candidate containing a foreground passive galaxy with a background emission-line galaxy. We indicate the emission and absorption lines at $z_{1}=0.128$ as red and magenta dashed lines, respectively, and the emission and absorption lines at $z_{2}=0.603$ as blue and cyan dot-dashed lines, respectively. We also note that this probable lens galaxy appears to be merging with another one in Figure 1(a).

The result from GLEE is shown in Figure 4. We model the lensed-arc feature in the masked region shown in the top-left panel. The predicted lensed arc of the best fitting model is shown in the top-right panel and the normalized image residuals are shown in the bottom-left panel. We can clearly see a single source in the bottom-right panel based on the lens model with $r_{\text {ein }}=0^{\prime \prime} .72 \pm_{0.02}^{0.04}$. The critical line (blue) and the caustics (red) predicted by our lens model are drawn in the top-right and bottom-right panels, respectively. Even though we have a sensible lens mass model of J084928+000949, we classify this object as a highly probably lens system rather than a definite lens. Spectroscopically there are two distinct redshifts identified. However, since the GAMA spectroscopy does not have sufficient spatial resolution and the foreground lens galaxy also shows emission lines, it is not fully clear whether the background source has multiply lensed images. For the purpose of lens modeling, we have decided to use certain bluish features as lensed images of the background source, but high-resolution imaging or spatially resolved spectroscopy is needed to unambiguously confirm this potential lens system.

Based on our current best-fit model of J084928+000949, we compute the total mass within the Einstein radius through $M_{\text {tot }}\left(<r_{\text {ein }}\right)=\pi\left(D_{\mathrm{L}} r_{\text {ein }}\right)^{2} \Sigma_{\mathrm{cr}}$, where $D_{\mathrm{L}}$ is the angular distance between us and the lens, and $\Sigma_{\mathrm{cr}}=c^{2} D_{\mathrm{L}} / 4 \pi G D_{\mathrm{LS}} D_{\mathrm{S}}$ is the critical surface mass density. The total mass for this highly probable lens system is $\log \left(M_{\text {tot }}\left(<r_{\text {ein }}\right) / M_{\odot}\right)=10.59 \pm_{0.02}^{0.05}$. We estimate the $\mathrm{AB}$ magnitudes in all grizy bands of the lens galaxy within a circular aperture of radius $r_{\text {ein }}$ : $m_{g}=20.66 \pm 0.02, m_{r}=19.53 \pm 0.02, m_{i}=18.98 \pm 0.02$, $m_{z}=18.57 \pm 0.03$, and $m_{v}=18.47 \pm 0.02$. With the same method in Oguri et al. (2014) using Bruzual \& Charlot (2003) stellar population synthesis model, we calculate the stellar mass $\log \left(M_{*} / M_{\odot}\right)=10.46 \pm 0.08$ including additional parameter on dust extinction at the galaxy redshift. The value is derived using Salpeter initial mass funcion (IMF). We note that we obtain similar stellar mass of $\log \left(M_{*} / M_{\odot}\right)=10.50 \pm 0.08$ even if we do not include the dust extinction. The stellar mass is consistent with the result from Taylor et al. (2011), $\log \left(M_{*} / M_{\odot}\right)=10.55 \pm 0.11$. From this analysis we can infer the dark matter fraction within the Einstein radius to be $\sim 25 \%$, which is comparable to those in SLACS and SL2S (Barnabè et al. 2011; Auger et al. 2010; Sonnenfeld et al. 2015).

\section{SUMMARY AND DISCUSSIONS}

Starting from the GAMA blended spectra catalog, we find 45 matches in HSC early data release S14A_0b. We use CHitah to separate lens light and lensed arc components. We identified 10 lens candidates via redshift information and morphology. To further confirm these lens candidates, we use GLEE to model the lensed arc components. After our examinations of the modeling results, we draw the following conclusions:

1. We identify 10 lens candidates via redshift information and morphology.

2. Through lens model fitting and examining the spectrum, we consider one system, HSC J084928+000949, as a highly probable lens. The Einstein radius $r_{\text {ein }}$ is around $0.72^{\prime \prime}$.

3. The highly probable lens is an interacting system which allows us to study the interplay between dark matter and stars.

4. For the highly probable lens, the dark matter fraction within $r_{\text {ein }}$ is $\sim 25 \%$.

Of the 14 strong-lens candidates from Holwerda et al. (2015) that have HSC imaging in the S14A_0b data release, we have classified 6 of them as probable lenses. The success rate of the GAMA's approach is thus roughly $\sim 50 \%$, although we note that further confirmation of these candidates is needed in order to obtain more robust estimates of the success rate.

After the first successful identification of a list of lens candidates including one highly probable lens system, we expect to find more strongly lensed galaxies in future data releases of HSC. Higher-resolution imaging (via, e.g., adaptive optics), or spatially resolved spectroscopy would be helpful to confirm further the nature of these candidates. The new interacting lens candidate is very valuable and with follow-up observation it would provide a great opportunity to study the interplay between dark matter and also the hierarchical formation/evolution of galaxies. 


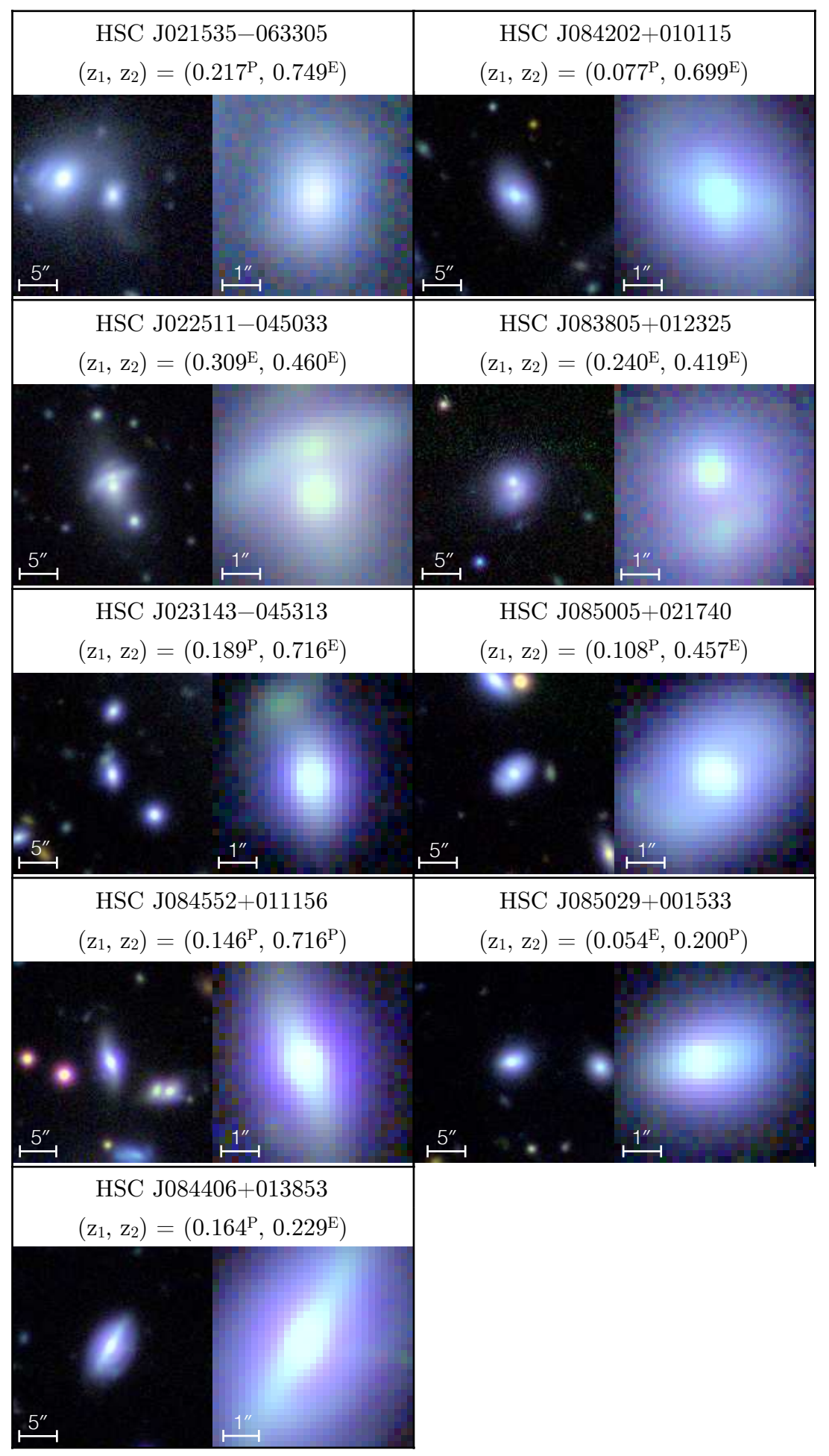

FIG. 2.- The nine probable lens candidates from the combination of GAMA spectroscopy and HSC SSP imaging. For each candidate, we show on the left a $25^{\prime \prime} \times 25^{\prime \prime}$ cutout and on the right a $5^{\prime \prime} \times 5^{\prime \prime}$ cutout of the riz color image. The spectral types of each candidate is denoted after the redshifts, where "P" stands for "passive galaxies" and "E" stands for "emission-line galaxies". J022511-045033 is a probable binary lens system. J084552+011156 and J084406+013853 are likely disky lenses. Some of them are low-redshift lenses, such as J084202+010115, J085005+021740 and J085029+001533. 
TABLE 1

The Catalog of Lens CANDidates SELECTED From GAMA in the HSC Survey

\begin{tabular}{lrrrc}
\hline HSC ID & GAMA ID & RA & DEC & $\left(z_{1}, z_{2}\right)$ \\
\hline HSC J084928+000949 $9^{\dagger}$ & 209222 & 132.36771 & 0.16360 & $\left(0.128^{\mathrm{P}}, 0.603^{\mathrm{E}}\right)$ \\
HSC J021535-063305 & 1126606 & 33.89513 & -6.55139 & $\left(0.217^{\mathrm{P}}, 0.749^{\mathrm{E}}\right)$ \\
HSC J022511-045033 & 1779869 & 36.29463 & -4.84248 & $\left(0.309^{\mathrm{E}}, 0.460^{\mathrm{E}}\right)$ \\
HSC J023143-045313 & 2005629 & 37.92875 & -4.88681 & $\left(0.189^{\mathrm{P}}, 0.716^{\mathrm{E}}\right)$ \\
HSC J084552+011156 & 300979 & 131.46746 & 1.19884 & $\left(0.146^{\mathrm{P}}, 0.716^{\mathrm{P}}\right)$ \\
HSC J084406+013853 & 323247 & 131.02333 & 1.64814 & $\left(0.164^{\mathrm{P}}, 0.229^{\mathrm{E}}\right)$ \\
HSC J084202+010115 & 371208 & 130.50925 & 1.02071 & $\left(0.077^{\mathrm{P}}, 0.699^{\mathrm{E}}\right)$ \\
HSC J083805+012325 & 375506 & 129.51933 & 1.39028 & $\left(0.240^{\mathrm{E}}, 0.419^{\mathrm{E}}\right)$ \\
HSC J085005+021740 & 417645 & 132.51888 & 2.29438 & $\left(0.108^{\mathrm{P}}, 0.457^{\mathrm{E}}\right)$ \\
HSC J085029+001533 & 599797 & 132.62096 & 0.25907 & $\left(0.054^{\mathrm{E}}, 0.200^{\mathrm{P}}\right)$ \\
\hline
\end{tabular}

$\S$ The strong lens candidate in GAMA blended spectra catalog. †The highly probable lens system via lens modeling. The Einstein radius is $r_{\text {ein }} \sim 0.72^{\prime \prime}$. The total mass within the Einstein radius is $M_{\text {tot }} \sim 10^{10.59} M_{\odot}$. The stellar mass is $M_{*} \sim 10^{10.46} M_{\odot}$, accounting for dust extinction. The remaining nine lens candidates without $\dagger$ cannot obtain reasonable lens models with GLEE, e.g., too small/large $r_{\text {ein }}$ or unphysical inverted source, due to the limited number of image pixels. The spectral types of each candidate is denoted after the redshifts, where "P" stands for "passive galaxies" and "E" stands for "emission-line galaxies".

HSC J084928+000949 (GAMA ID: 209222)

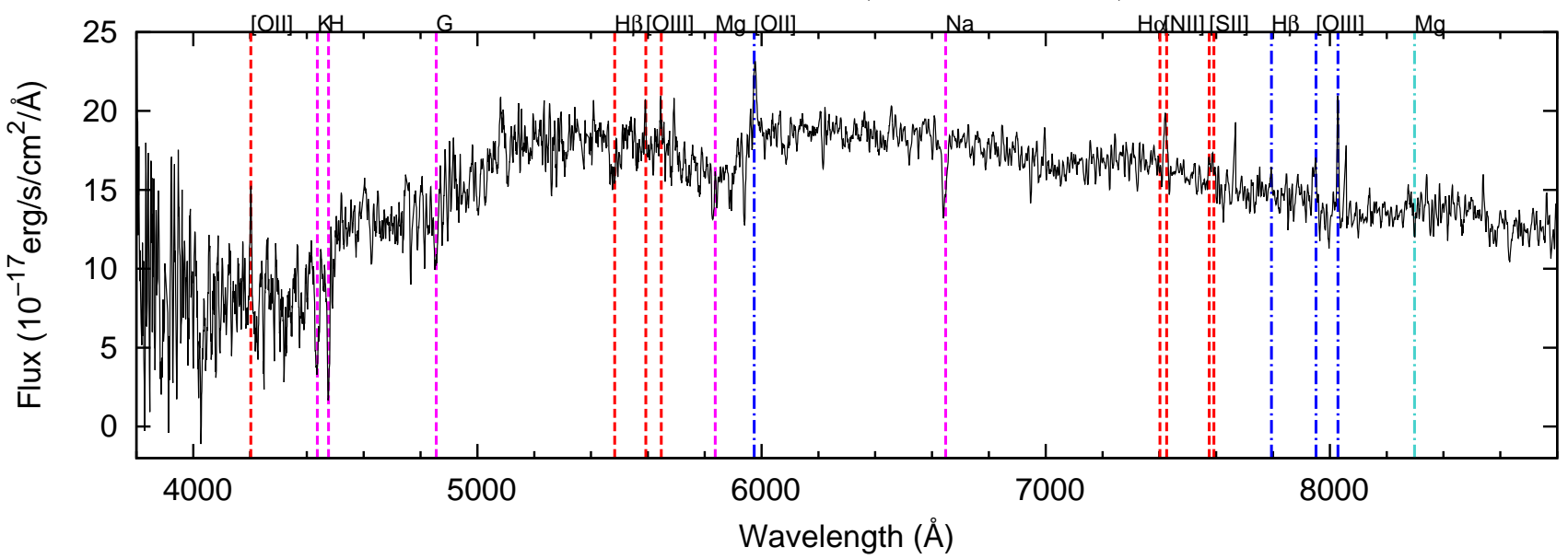

FIG. 3. - Blended spectra of HSC J084928+000949 (black line) from the GAMA survey (GAMA ID: 209222). Red and magenta dashed lines show the emission and absorption lines at $z_{1}=0.128$ respectively. Blue and cyan dot-dashed lines show the emission and absorption lines at $z_{2}=0.603$ respectively. http://www.gama/survey.org/dr2/data/spectra/gama/reduced_08/1d/G09_Y1_CS2_288.fit 

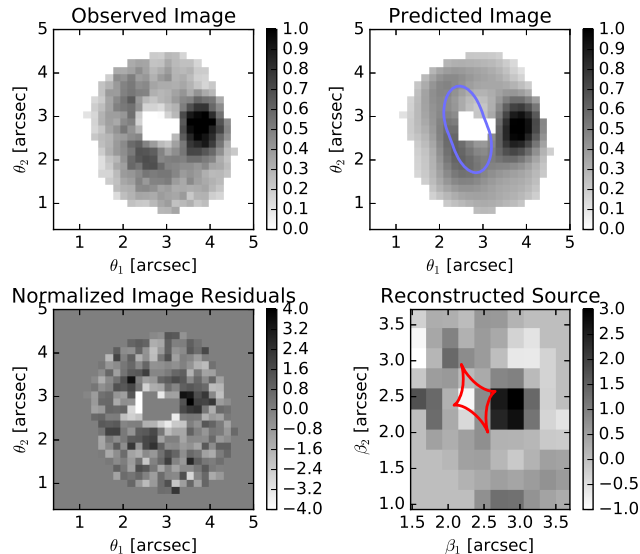

FIG. 4.- The result from lens modeling of the highly probable lens HSC J084928+000949. We model the lensed-arc feature in the masked region shown in the top-left panel. The predicted lensedarc of the best-fitting model is shown in the top-right panel. The normalized image residuals are shown in the bottom-left panel. We can see clearly a compact source galaxy in the bottom-right panel. The critical line (blue) and the caustics (red) predicted by our lens model are drawn in the top-right and bottom-right panels, respectively.

\section{ACKNOWLEDGMENTS}

We thank Ying-Tung Chen and Li-Hwai Lin for useful discussions, and the anonymous referee for helpful comments. J.H.H.C. would like to thank ChihFan Chen and Kenneth Wong for algorithm support. J.H.H.C. and S.H.S. gratefully acknowledge support by the Ministry of Science and Technology in Taiwan via grant MOST-103-2112-M-001-003-MY3, and support by the Max Planck Society through the Max Planck Research Group for S.H.S. T.C. acknowledges the Ministry of Science and Technology in Taiwan via grant MOST103-2112-M-002-020-MY3. A.M. is supported by World Premier International Research Center Initiative (WPIInitiative), MEXT, Japan, and also acknowledges the support of the Japan Society for Promotion of Science (JSPS) fellowship. M.O. acknowledges support in part by World Premier International Research Center Initiative (WPI Initiative), MEXT, Japan, and Grant-in-Aid for Scientific Research from the JSPS (26800093). The Hyper Suprime-Cam (HSC) collaboration includes the astronomical communities of Japan and Taiwan, and Princeton University. The HSC instrumentation and software were developed by the National Astronomical Observatory of Japan (NAOJ), the Kavli Institute for the Physics and Mathematics of the Universe (Kavli IPMU), the University of Tokyo, the High Energy Accelerator Research Organization (KEK), the Academia Sinica Institute for Astronomy and Astrophysics in Taiwan (ASIAA), and Princeton University. Funding was contributed by the FIRST program from Japanese Cabinet Office, the Ministry of Education, Culture, Sports, Science and Technology (MEXT), the Japan Society for the Promotion of Science (JSPS), Japan Science and Technology Agency (JST), the Toray Science Foundation, NAOJ, Kavli IPMU, KEK, ASIAA, and Princeton University. This paper makes use of software developed for the Large Synoptic Survey Telescope. We thank the LSST Project for making their code available as free software at http://dm.lsstcorp.org. The Pan-STARRS1 Surveys (PS1) have been made possible through contributions of the Institute for Astronomy, the University of Hawaii, the Pan-STARRS Project Office, the Max-Planck Society and its participating institutes, the Max Planck Institute for Astronomy, Heidelberg and the Max Planck Institute for Extraterrestrial Physics, Garching, The Johns Hopkins University, Durham University, the University of Edinburgh, Queen's University Belfast, the Harvard-Smithsonian Center for Astrophysics, the Las Cumbres Observatory Global Telescope Network Incorporated, the National Central University of Taiwan, the Space Telescope Science Institute, the National Aeronautics and Space Administration under Grant No. NNX08AR22G issued through the Planetary Science Division of the NASA Science Mission Directorate, the National Science Foundation under Grant No. AST-1238877, the University of Maryland, and Eotvos Lorand University (ELTE).

\section{APPENDIX}

\section{THE POSSIBLE LENS CANDIDATES FROM GAMA IN THE HSC SURVEY}

We classify the remaining 33 objects as possible lens candidates or non-lenses (e.g., galaxy pairs), and list them in Table 2 and Figure 5. Possible reasons are included in Table 2. We mark 8 objects by $\S$ that are previously identified as strong lens candidates by Holwerda et al. (2015), i.e., objects with blended GAMA spectra of foreground passive galaxies with background emission-line galaxy. For those object with $\vartheta_{\text {sep }}<1.0$, higher resolution imaging from space or adaptive optics are needed to detect lensed features.

\section{REFERENCES}

Auger, M. W., Treu, T., Bolton, A. S., et al. 2010, ApJ, 724, 511

Axelrod, T., Kantor, J., Lupton, R. H., \& Pierfederici, F. 2010, in Society of Photo-Optical Instrumentation Engineers (SPIE) Conference Series, Vol. 7740, Society of Photo-Optical Instrumentation Engineers (SPIE) Conference Series, 15

Baldry, I. K., Robotham, A. S. G., Hill, D. T., et al. 2010, MNRAS, 404, 86

Baldry, I. K., Alpaslan, M., Bauer, A. E., et al. 2014, MNRAS, 441,2440

Barnabè, M., Czoske, O., Koopmans, L. V. E., Treu, T., \& Bolton, A. S. 2011, MNRAS, 415, 2215

Bolton, A. S., Burles, S., Koopmans, L. V. E., Treu, T., \& Moustakas, L. A. 2006, ApJ, 638, 703
Bolton, A. S., Brownstein, J. R., Kochanek, C. S., et al. 2012, ArXiv e-prints (1201.2988)

Brownstein, J. R., Bolton, A. S., Schlegel, D. J., et al. 2012, ApJ, 744,41

Bruzual, G., \& Charlot, S. 2003, MNRAS, 344, 1000

Cabanac, R. A., Alard, C., Dantel-Fort, M., et al. 2007, A\&A, 461,813

Chan, J. H. H., Suyu, S. H., Chiueh, T., et al. 2015, ApJ, 807, 138

Driver, S. P., Norberg, P., Baldry, I. K., et al. 2009, Astronomy and Geophysics, 50,12

Driver, S. P., Hill, D. T., Kelvin, L. S., et al. 2011, MNRAS, 413, 971

Gavazzi, R., Treu, T., Marshall, P. J., Brault, F., \& Ruff, A. 2012, ApJ, 761, 170 


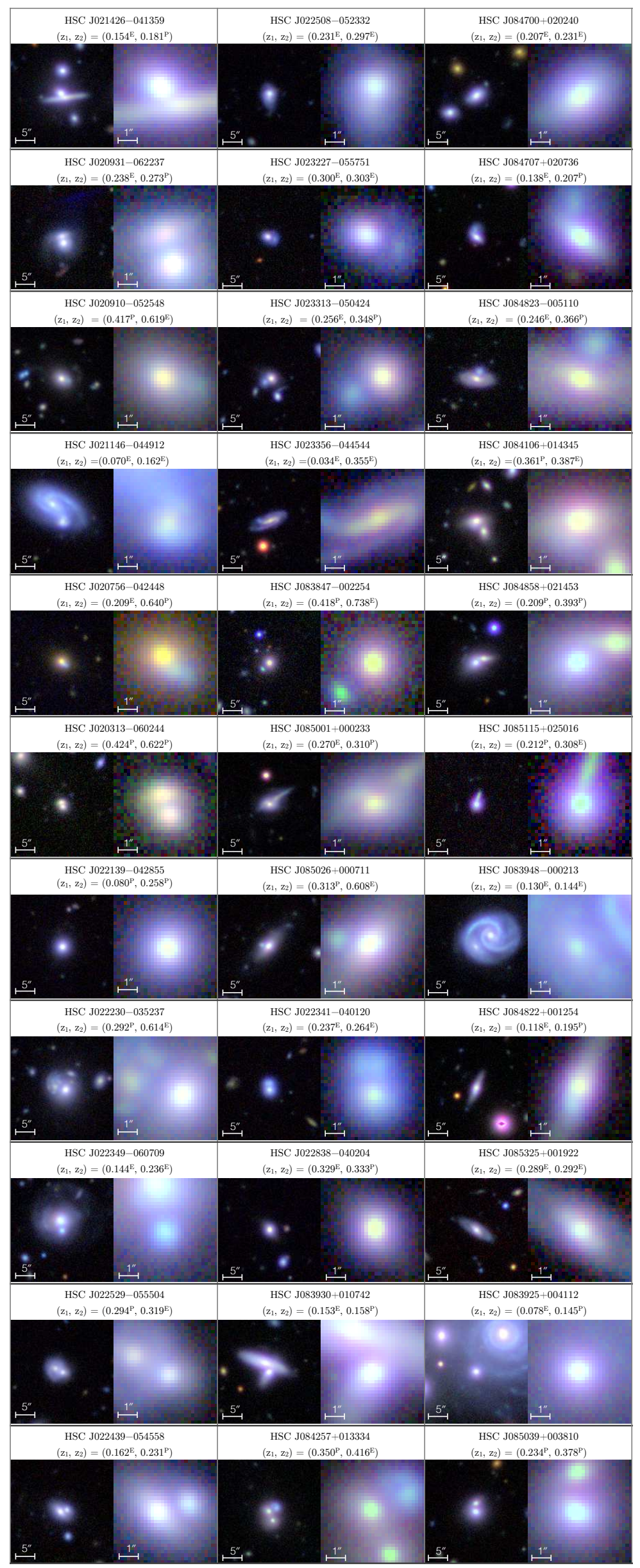

FIG. 5. - The remaining candidates from the combination of GAMA spectroscopy and HSC SSP imaging. For each candidate, we show

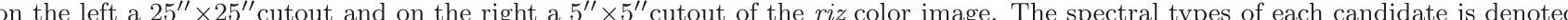


TABLE 2

The Catalog of the possible lens Candidates or NON-Lenses from GAMA in the HSC SURVeY

\begin{tabular}{|c|c|c|c|c|c|}
\hline HSC ID & GAMA ID & $\mathrm{RA}$ & $\overline{\mathrm{DEC}}$ & $\left(z_{1}, z_{2}\right)$ & possible reason \\
\hline HSC J021426-041359 & 1217811 & 33.60950 & -4.23305 & $\left(0.154^{\mathrm{E}}, 0.181^{\mathrm{P}}\right)$ & $\vartheta_{\text {sep }}<1.0^{\prime \prime}$ \\
\hline HSC J020931-062237 & 1298084 & 32.37917 & -6.37684 & $\left(0.238^{\mathrm{E}}, 0.273^{\mathrm{P}}\right)$ & $\vartheta_{\text {sep }}<1.0^{\prime \prime}$ \\
\hline HSC J020910-052548§ & 1312058 & 32.29279 & -5.43011 & $\left(0.417^{\mathrm{P}}, 0.619^{\mathrm{E}}\right)$ & no visible strong lensing feature $\ddagger$ \\
\hline HSC J021146-044912 & 1320592 & 32.94087 & -4.82006 & $\left(0.070^{\mathrm{E}}, 0.162^{\mathrm{E}}\right)$ & galaxy pair \\
\hline HSC J020756-042448 & 1440776 & 31.98304 & -4.41341 & $\left(0.209^{\mathrm{E}}, 0.640^{\mathrm{P}}\right)$ & galaxy pair \\
\hline HSC J020313-060244 & 1537351 & 30.80412 & -6.04563 & $\left(0.424^{\mathrm{P}}, 0.622^{\mathrm{P}}\right)$ & galaxy pair \\
\hline HSC J022139-042855 & 1675035 & 35.41175 & -4.48197 & $\left(0.080^{\mathrm{E}}, 0.258^{\mathrm{P}}\right)$ & no resolvable strong lensing feature $\ddagger$ \\
\hline HSC J022230-035237§ & 1684064 & 35.62492 & -3.87688 & $\left(0.292^{\mathrm{P}}, 0.614^{\mathrm{E}}\right)$ & multiple objects \\
\hline HSC J022349-060709 & 1760310 & 35.95496 & -6.11922 & $\left(0.144^{\mathrm{E}}, 0.236^{\mathrm{E}}\right)$ & galaxy pair \\
\hline HSC J022529-055504§ & 1763319 & 36.37075 & -5.91766 & $\left(0.294^{\mathrm{P}}, 0.319^{\mathrm{E}}\right)$ & $\vartheta_{\text {sep }}<1.0^{\prime \prime}$ \\
\hline HSC J022439-054558 & 1765570 & 36.16213 & -5.76612 & $\left(0.162^{\mathrm{E}}, 0.231^{\mathrm{P}}\right)$ & galaxy pair \\
\hline HSC J022508-052332 & 1771132 & 36.28483 & -5.39210 & $\left(0.231^{\mathrm{E}}, 0.297^{\mathrm{E}}\right)$ & $\vartheta_{\mathrm{sep}}<1.0^{\prime \prime}$ \\
\hline HSC J023227-055751 & 1988308 & 38.11142 & -5.96424 & $\left(0.300^{\mathrm{E}}, 0.303^{\mathrm{E}}\right)$ & $\vartheta_{\text {sep }}<1.0^{\prime \prime}$ \\
\hline HSC J023313-050424 & 2002618 & 38.30588 & -5.07329 & $\left(0.256^{\mathrm{E}}, 0.348^{\mathrm{P}}\right)$ & $\vartheta_{\text {sep }}<1.0^{\prime \prime}$ \\
\hline HSC J023356-044544 & 2007752 & 38.48246 & -4.76219 & $\left(0.034^{\mathrm{E}}, 0.355^{\mathrm{E}}\right)$ & no counter image $\ddagger$ \\
\hline HSC J083847-002254§ & 202448 & 129.69546 & -0.38179 & $\left(0.418^{\mathrm{P}}, 0.738^{\mathrm{E}}\right)$ & galaxy pair \\
\hline HSC J085001+000233 & 209263 & 132.50596 & 0.04250 & $\left(0.270^{\mathrm{E}}, 0.310^{\mathrm{P}}\right)$ & $\vartheta_{\mathrm{sep}}<1.0^{\prime \prime}$ \\
\hline HSC J085026+000711§ & 209295 & 132.61013 & 0.11972 & $\left(0.313^{\mathrm{P}}, 0.608^{\mathrm{E}}\right)$ & galaxy pair \\
\hline HSC J022341-040120 & 2308869 & 35.92083 & -4.02211 & $\left(0.237^{\mathrm{E}}, 0.264^{\mathrm{E}}\right)$ & $\vartheta_{\mathrm{sep}}<1.0^{\prime \prime}$ \\
\hline HSC J022838-040204 & 2379807 & 37.16025 & -4.03447 & $\left(0.329^{\mathrm{E}}, 0.333^{\mathrm{P}}\right)$ & $\vartheta_{\text {sep }}<1.0^{\prime \prime}$ \\
\hline HSC J083930+010742 & 300500 & 129.87425 & 1.12844 & $\left(0.153^{\mathrm{E}}, 0.158^{\mathrm{P}}\right)$ & $\vartheta_{\text {sep }}<1.0^{\prime \prime}$ \\
\hline HSC J084257+013334§ & 323200 & 130.73717 & 1.55957 & $\left(0.350^{\mathrm{P}}, 0.416^{\mathrm{E}}\right)$ & $\vartheta_{\text {sep }}<1.0^{\prime \prime}$ \\
\hline HSC J084700+020240 & 345974 & 131.75004 & 2.04437 & $\left(0.207^{\mathrm{E}}, 0.231^{\mathrm{E}}\right)$ & $\vartheta_{\text {sep }}<1.0^{\prime \prime}$ \\
\hline HSC J084707+020736 & 345984 & 131.78108 & 2.12672 & $\left(0.138^{\mathrm{E}}, 0.207^{\mathrm{P}}\right)$ & galaxy pair \\
\hline HSC J084823-005110 & 3624490 & 132.09767 & -0.85274 & $\left(0.246^{\mathrm{E}}, 0.366^{\mathrm{P}}\right)$ & galaxy pair \\
\hline HSC J084106+014345§ & 380839 & 130.27629 & 1.72911 & $\left(0.361^{\mathrm{P}}, 0.387^{\mathrm{E}}\right)$ & $\vartheta_{\text {sep }}<1.0^{\prime \prime}$ \\
\hline HSC J084858+021453 & 386427 & 132.24292 & 2.24817 & $\left(0.209^{\mathrm{P}}, 0.393^{\mathrm{P}}\right)$ & galaxy pair \\
\hline HSC J085115+025016 & 422882 & 132.81062 & 2.83784 & $\left(0.212^{\mathrm{P}}, 0.308^{\mathrm{E}}\right)$ & galaxy pair \\
\hline HSC J083948-000213 & 573744 & 129.95100 & -0.03694 & $\left(0.130^{\mathrm{E}}, 0.144^{\mathrm{E}}\right)$ & $\vartheta_{\text {sep }}<1.0^{\prime \prime}$ \\
\hline HSC J084822+001254 & 599598 & 132.08975 & 0.21503 & $\left(0.118^{\mathrm{E}}, 0.195^{\mathrm{P}}\right)$ & no counter image $\ddagger$ \\
\hline HSC J085325+001922 & 599995 & 133.35229 & 0.32283 & $\left(0.289^{\mathrm{E}}, 0.292^{\mathrm{E}}\right)$ & $\vartheta_{\text {sep }}<1.0^{\prime \prime}$ \\
\hline HSC J083925+004112 & 621991 & 129.85479 & 0.68659 & $\left(0.078^{\mathrm{E}}, 0.145^{\mathrm{P}}\right)$ & no visible strong lensing feature $\ddagger$ \\
\hline HSC J085039+003810 & 622326 & 132.66296 & 0.63611 & $\left(0.234^{\mathrm{P}}, 0.378^{\mathrm{P}}\right)$ & galaxy pair \\
\hline
\end{tabular}

$\S$ Strong-lens candidates in GAMA blended spectra catalog. ‡We inspect for strong lensing features using the "lensed images" based on color separation by СНІтАн. We predict $\vartheta_{\text {sep }}$ with a fiducial velocity dispersion of $\sigma \sim 250 \mathrm{~km} / \mathrm{s}$. The spectral types of each candidate

is denoted after the redshifts, where "P" stands for "passive galaxies" and "E" stands for "emission-line galaxies". 
González-Nuevo, J., Lapi, A., Fleuren, S., et al. 2012, ApJ, 749, 65

Hezaveh, Y. D., Marrone, D. P., Fassnacht, C. D., et al. 2013, ApJ, 767, 132

Holwerda, B. W., Keel, W. C., \& Bolton, A. 2007, AJ, 134, 2385

Holwerda, B. W., Baldry, I. K., Alpaslan, M., et al. 2015, MNRAS, 449,4277

Hopkins, A. M., Driver, S. P., Brough, S., et al. 2013, MNRAS, 430,2047

Hopkins, P. F., Somerville, R. S., Hernquist, L., et al. 2006, ApJ, 652,864

Ivezic, Z., Tyson, J. A., Abel, B., et al. 2008, ArXiv:0805.2366

Kassiola, A., \& Kovner, I. 1993, ApJ, 417, 450

Keel, W. C., Manning, A. M., Holwerda, B. W., et al. 2013, PASP, 125,2

Liske, J., Baldry, I. K., Driver, S. P., et al. 2015, MNRAS, 452, 2087

Lotz, J. M., Jonsson, P., Cox, T. J., \& Primack, J. R. 2008, MNRAS, 391, 1137

Magnier, E. A., Schlafly, E., Finkbeiner, D., et al. 2013, ApJS, 205, 20

Marshall, P. J., Hogg, D. W., Moustakas, L. A., et al. 2009, ApJ, 694,924

Miyazaki, S., Komiyama, Y., Sekiguchi, M., et al. 2002, PASJ, 54 833
Miyazaki, S., Komiyama, Y., Nakaya, H., et al. 2012, in Society of Photo-Optical Instrumentation Engineers (SPIE) Conference Series, Vol. 8446, Society of Photo-Optical Instrumentation Engineers (SPIE) Conference Series, 0

More, A., Cabanac, R., More, S., et al. 2012, ApJ, 749, 38

Negrello, M., Hopwood, R., De Zotti, G., et al. 2010, Science, 330, 800

Oguri, M., Rusu, C. E., \& Falco, E. E. 2014, MNRAS, 439, 2494

Schlafly, E. F., Finkbeiner, D. P., Jurić, M., et al. 2012, ApJ, 756, 158

Sérsic, J. L. 1963, Boletin de la Asociacion Argentina de Astronomia La Plata Argentina, 6, 41

Sonnenfeld, A., Gavazzi, R., Suyu, S. H., Treu, T., \& Marshall, P. J. 2013a, ApJ, 777, 97

Sonnenfeld, A., Treu, T., Gavazzi, R., et al. 2013b, ApJ, 777, 98

Sonnenfeld, A.. Treu, T., Marshall, P. J., et al. 2015, ApJ, 800, 94 Suyu, S. H., \& Halkola, A. 2010, A\&A, 524, A94

Suyu, S. H., Hensel, S. W., McKean, J. P., et al. 2012, ApJ, 750 10

Taylor, E. N., Hopkins, A. M., Baldry, I. K., et al. 2011, MNRAS, 418,1587

Tonry, J. L., Stubbs, C. W., Lykke, K. R., et al. 2012, ApJ, 750, 99

Vieira, J. D., Marrone, D. P., Chapman, S. C., et al. 2013, Nature, 495, 344 